\title{
94. Über das Haarsche Mass in der lokal bikompakten Gruppe.
}

\author{
Von Shizuo KaKUTANI. \\ Mathematisches Institut der Kaiserlichen Universität, Osaka. \\ Kunihiko KodarRa. \\ Physikalisches Institut der Kaiserlichen Universität, Tokyo. \\ (Comm. by T. Takagi, M.I.A., July 12, 1944.)
}

Zur Definition des Haarschen Masses $m$ in einer lokal bikompakten, nicht separablen Gruppe gibt es zwei Möglichkeiten. Nach der ersten gewöhnlichen Definition wird $m$ zunächst für alle Borelschen Mengen erklärt und dann zum vollständigen Mass vervollständigt; nach der zweiten wird dagegen $m$ zunächst nur für die Mengen mit Baireschen charakteristischen Funktionen - wir wollen solche Menge Bairesch nennen ${ }^{1}$ - definiert und dann vervollständigt. Sind nun diese zwei Definitionen äquivalent ?' In der vorliegenden Note soll diese Frage bejahend beantwortet werden ${ }^{3)}$. Dabei benutzen wir die „Quasi-separabilität " der lokal bikompakten Gruppe, d. h. dass jede solche Gruppe als eine separable betrachtet werden kann, solange man mit höchstens abzählbar vielen Baireschen Funktionen zu tun hat. (Für den genauen Sinn dieser Behauptung siehe unten, § 3.)

$\S 1$. Borelsche und Bairesche Mengen. Es sei $\Omega$ ein lokal bikompakter Raum mit der Eigenschaft: $\Omega$ lässt sich als Summe höchstens abzählbar vieler bikompakter Teilmengen $\Omega_{l_{c}}$ darstellen:

$$
\Omega=\sum_{k=1}^{\infty} \Omega_{k}, \quad \Omega_{k}: \text { bikompakt. }
$$

Eine Teilmenge $B$ aus $\Omega$ heisst bekanntlich Borelsch, wenn sie zum alle offenen Mengen enthaltenden minimalen Borelschen Mengenkörper gehört. Wir bezeichnen mit $\mathfrak{B}(\Omega)$ die Familie aller Borelschen Teilmengen von 2 . Andererseits nennen wir eine Familie $\mathfrak{f}$ der (reellen) Funktionen $f(p)$ auf $\Omega$ Bairesch, wenn i) f ein Ring im algebraischen Sinne ist und 1 (die Funktion mit dem konstanten Wert 1) enthält, und ii) aus $f(p)=\lim _{k \rightarrow \infty} f_{k}(p), f_{k}(p) \in \mathfrak{f}$ die Relation $f(p) \in \mathfrak{f}$ folgt. Eine Funktion $f(p)$ aus $\Omega$ heisst Bairesch, wenn sie zur alle stetigen Funktionen enthaltenden minimalen Baireschen Familie gehört.

Definition 1. Eine Teilmenge $B \subset \Omega$ heisst Bairesch, wenn ihre charakteristische Funktion $c_{B}(p)$ Bairesch ist.

1) Vgl. K. Kodaira: [1] Über die Gruppe der messbaren Abbildungen, diese Proc. 17 (1941), 18-23; [2] Über die Beziehung zwischen den Massen und den Topologien in einer Gruppe, Proc. Phys.-Math. Soc. Japan, 23 (1941), 67-119, Kap. II. In diesen beiden Arbeiten haben wir die Menge mit Bairescher charakteristischer Funktion „Borelsch “ genannt.

2) Vgl. K. Kodaira: [2], S. 80.

3) Für die "Torusgruppe " ist dieses Problem von S. Kakutani gelöst. Vgl. S. Kakutani : [1] Notes on infinite product measure spaces, II. Proc. 19 (1943), 184-188. 
Die Familie aller Baireschen Teilmengen von $\Omega$ sei mit $\mathfrak{b}(\Omega)$ bezeichnet. Es ist offenbar

$$
\mathfrak{b}(\Omega) \leqq \mathfrak{B}(\Omega),
$$

die jede stetige, und also jede Bairesche Funktion $\mathfrak{B}(\Omega)$-messbar ist. $\Omega$ ist bekanntlich ein vollständig regulärer Raum. $\Omega$ besitzt also ein Umgebungssystem $\{U(p)\}$, das lauter aus Baireschen offenen Mengen besteht. Umgekehrt sei ein solcher Borelkörper $\Re$ gegeben, der irgendein Umgebungssystem $\{V(p)\}$ von $\Omega$ enthält. Dann ist jede stetige Funktion auf $\Omega \Re$-messbar, also muss $\mathfrak{b}(\Omega) \leqq \Re$ sein. Es gilt also der

Satz 1. Unter allen Borelkörpern, die mindestens eine Basis (d.h. Umgebungssystem) enthalten, ist $\mathfrak{b}(\Omega)$ der minimale ${ }^{1)}$.

Bezeichnet man für die gegebene Familie der Funktionen $\mathfrak{F}$ die $\mathfrak{F}$ enthaltende minimale Bairesche Familie mit $\mathrm{f}(\mathfrak{F})$ so gilt allgemein

$$
\mathfrak{f}(\mathfrak{F})=\sum \mathfrak{f}\left(f_{1}, f_{2}, \ldots, f_{k}, \ldots\right), \quad f_{k} \in \mathfrak{F},
$$

d. h. $f(\mathfrak{F})$ ist die Vereinigungsmenge aller Baireschen Familien, die aus abzählbar vielen Funktionen aus $\mathfrak{F}$ erzeugt werden. Insbesondere wird also jede Bairesche Funktion $f$ auf $\Omega$ in einem $f\left(\varphi_{1}, \varphi_{2}, \ldots\right)$ enthalten, wo $\varphi_{1}, \varphi_{2}, \ldots$ stetige Funktionen sind.

Nun betrachten wir ein gegebenes System $\left\{\varphi_{1}, \varphi_{2}, \ldots\right\}$ der abzählbar vielen stetigen Funktionen $\varphi_{1}, \varphi_{2}, \ldots$. Wegen der Bedingung (a) gibt es eine auf $\Omega$ definierte überall stetige positive Funktion $\Psi(p)$ mit der Eigenschaft: die Menge $\{p ; \Psi(p) \leqq c\}$ ist für beliebiges $c>0$ immer bikompakt. Definiert man dann die Quasi-metrik $\rho(p, q)$ durch

$$
\rho(p, q)=|\Psi(p)-\Psi(q)|+\sum_{k=1}^{\infty} \frac{\left|\varphi_{k}(p)-\varphi_{k}(q)\right|}{2^{k}\left(1+\left|\varphi_{k}(p)-\varphi_{k}(q)\right|\right)}
$$

und identifiziert zwei Punkte $p, q$ mit $\rho(p, q)=0$, so erhält man einen lokal kompakten separablen metrischen Raum $\bar{\Omega}$. $\bar{\Omega}$ ist offenbar ein Zerlegungsraum von $\Omega$, und für kompakte Teilmenge $\bar{F} \subset \bar{\Omega}$ ist die entsprechende Teilmenge $F \subset \Omega$ auch bikompakt. $\varphi_{k}(p)$ kann dabei offenbar als eine stetige Funktion auf $\overline{\boldsymbol{\Omega}}$ aufgefasst werden. Es gilt also der

Satz 2. Es sei eine aus höchstens abzählbar vielen Baireschen Funktionen erzeugte Bairesche Familie $f\left(f_{1}, f_{2}, \ldots\right)$ gegeben. Dann gibt es einen lokal kompakten separablen metrischen Zerlegungsraum $\bar{\Omega}$ von $\Omega$ mit der Eigenschaft: für kompakte Teilmenge $\bar{F} \subset \bar{\Omega}$ ist die entsprechende $F \subset \Omega$ auch bikompakt, und jede Funktion aus $f\left(f_{1}, f_{2}, \ldots\right)$ lässt sich als eine Funktion auf $\bar{\Omega}$ auffassen.

Jede Auissage über die Baireschen Funktionen, die für jeden lokal kompakten separablen metrischen Raum richtig ist, gilt also auch für den allgemeinen lokal bikompakten Raum, solange sie sich auf höchstens abzählbar viele Bairesche Funktionen bezieht. Z. B. ist jede Bairesche offene Menge ein $F_{\sigma}$, jede Bairesche abgeschlossene Menge ein $G_{\dot{o}}$.

1) K. Kodaira : [1], Satz 5. 
§2. Lebesguesches Mass in lokal bikompakten Räumen.

Definition 2. Ein in $\Omega$ erklärtes Carathéodorysches äusseres Mass $\mu^{*}$ heisst Lebesguesches Mass erster Art (kurz: LI-Mass), wenn i) jede Borelsche Menge $\mu^{*}$-messbar ist, ii) für bikompaktes $A \mu^{*}(A)<+\infty$ ist und iii) für jede Teilmenge $A \subset \Omega \mu^{*}(A)$ die untere Grenze aller Mass $\mu^{*}(U)$ von $A$ enthaltenden offenen Mengen $U$ ist: $\mu^{*}(A)=\inf _{U \rightarrow A} \mu^{*}(U)$, $U$ : offen.

Definition $2_{\text {II. }}$ Ein in $\Omega$ erklärtes Carathéodorysches äusseres Mass $m^{*}$ heisst Lebesguesches Mass zweiter Art (kurz: LII-Mass), wenn i) jede Bairesche Menge $m^{*}$-messbar ist, ii) für bikompaktes $A$ $m^{*}(A)<+\infty$ ist und iii) für jedes $A \subset \Omega m^{*}(A)$ die untere Grenze aller Masse $m^{*}(U)$ von $A$ enthaltenden Baireschen offenen Mengen $U$ ist: $m^{*}(A)=\inf _{U>A} m^{*}(U), U:$ Bairesch offen.

Für $\mu^{*}$-bzw. $m^{*}$-messbares $A$ schreiben wir ferner $\mu(A)$ bzw. $m(A)$ statt $\mu^{*}(A)$ bzw. $m^{*}(A)$. Die Bedingung iii) in der Definition $2_{\mathrm{II}}$ lässt sich durch die folgende schwächere ersetzen:

iii)*

$$
m^{*}(A)=\inf _{B>A} m^{*}(B), \quad B \in \mathfrak{b}(\Omega) ;
$$

$m^{*}$ ist also in der Terminologie in einer früheren Arbeit ${ }^{1)}$ von einem der Verfasser nichts anderes als das zur Topologie von $\Omega$ gehörige Mass.

Wir bezeichnen mit $(C)$ die Familie aller solchen stetigen Funktionen $\varphi(p)$, für welche die Menge $\{p ; \varphi(p) \neq 0\}$ relativ bikompakt ist. Für ein $L I$-Mass $\mu^{*}$ bzw. LII-Mass $m^{*}$ ist

$$
I(\varphi)=\int_{\Omega} \varphi(p) \mu(d p) \quad \text { bzw. } \int_{\Omega} \varphi(p) m(d p)
$$

ein auf $(C)$ erklärtes lineares Funktional, und sogar ein positives. Umgekehrt gilt der

Satz 3. Jedes auf $(C)$ erklärte positive lineare Funktional $I(\varphi)$ lässt sich mit einem LI-Mass $\mu^{*}$ so darstellen:

$$
I(\varphi)=\int_{\Omega} \varphi(p) \mu(d p)
$$

$\mu^{*}$ wird dabei durch $I(\varphi)$ eindeutig bestimmt ${ }^{2}$.

Ist nun ein $L I$-Mass $\mu^{*}$ gegeben, so wird daraus durch

$$
m^{*}(A)=\inf _{B>A} \mu(B), \quad B \in \mathfrak{b}(\Omega)
$$

ein $L I I$-Mass $m^{*}$ abgeleitet. $\mu^{*}$ ist dabei offenbar eine Erweiterung von $m^{*}$; das bedeutet, dass jedes $m^{*}$-messbare $A$ auch $\mu^{*}$-messbar ist und $\mu(A)=m(A)$ gilt. Umgekehrt lässt sich jedes $L I I$-Mass $m^{*}$ mittels der Relation

$$
I(\varphi)=\int_{\Omega} \varphi(p) m(d p)=\int_{\Omega} \varphi(p) \mu(d p)
$$

1) Vgl. K. Kodaira: [1], Definition 5.

2) Wir übergehen hier den einfachen Beweis dieses Satzes. 
auf das LI-Mass $\mu^{*}$ erweitern. LI- und LII-Mass beziehen sich also miteinander ein-eindeutig ${ }^{1)}$.

Nun betrachten wir den Produktraum $\Omega \times \Omega$. Den minimalen Borelkörper, der $\{A \times B ; A, B \in \mathfrak{b}(\Omega)\}$ enthält, bezeichnen wir mit $\mathfrak{b}(\Omega) \times \mathfrak{b}(\Omega)$. Da dieser Borelkörper $\mathfrak{b}(\Omega) \times \mathfrak{b}(\Omega)$ offenbar eine Basis von $\Omega \times \Omega$ enthält, so gilt wegen des Satzes 1 der

Satz 4. Es ist $\mathfrak{b}(\Omega) \times \mathfrak{b}(\Omega)=\mathfrak{b}(\Omega \times \Omega)$.

Für LII-Mass $m^{*}$ gilt also der Fubinische Satz.

§3. Quasi-separabilität der lokal bikompakten Gruppe. Es sei $G$ eine lokal bikompakte Gruppe mit der Eigenschaft (a); die Elementen von $G$ seien mit $a, s, t \ldots$ bezeichnet. Bekanntlich gibt es in $G$ ein und bis auf multiplikative Konstante nur ein links-invariantes Lebesguesches Mass erster bzw. zweiter Art ${ }^{2}$.

Definition 3. Das Haarsche Mass $m^{*}$ von $G$ ist das in $G$ erklärte links-invariante Lebesguesche Mass zweiter Art.

Es sei $\mathfrak{S}$ der Hilbertsche Raum aller $m^{*}$-quadrat-summierbaren Funktionen auf $G$, und $\boldsymbol{U}$ die Gruppe aller unitären Operatoren auf ). Die Norm der Funktion $f \in \mathfrak{S}$ sei mit $\|f\|$ bezeichnet. Ferner sei $\boldsymbol{U}$ nach der starken Topologie topologisiert. Der Translationsoperator $T_{a}: f(t) \rightarrow f\left(a^{-1} t\right)$ ist unitär, und die Abbildung $a \rightarrow T_{a}$ von $G$ in $U$ ist umkehrbar eindeutig und beiderseitig stetig. $G$ wird also durch die unitären Transformationen $T_{a}$ topologisch isomorph dargestellt ${ }^{3)}$.

Satz 5. Für jedes $f \in \mathfrak{L}$ ist die durch alle $T_{a} f$ aufgespannte abgeschlossene lineare Mannigfaltigkeit $\left[T_{a} f ; a \in G\right]$ separabel ${ }^{4}$.

Beweis. $f\left(s^{-1} t\right)$ ist eine $m^{*}$-messbare Funktion von $t \times s \in G \times G^{5)}$. $G$ lässt sich nach der Voraussetzung als eine Summe höchstens abzählbar vieler $m^{*}$-messbarer Teilmengen $A_{k}$ mit $m\left(A_{k}\right)<+\infty$ darstellen. In jedem $G \times A_{k}$ ist $f\left(s^{-1} t\right) m^{*}$-quadrat-summierbar. Man kann also $g_{j k}^{(\nu)} \in \mathfrak{S}$ und beschränkte $m^{*}$-messbare Funktionen $h_{j k}^{(\nu)}$ so wählen, dass

$$
\lim _{\nu \rightarrow \infty} \iint_{G \times A_{k}}\left|f\left(s^{-1} t\right)-\sum_{j=1}^{J(\nu)} h_{j k}^{(\nu)}(s) g_{j k}^{(\nu)}(t)\right|^{2} m(d t) m(d s)=0
$$

gilt. Hieraus folgt nach dem Satz von Fubini ${ }^{6)}$

$$
\int_{A_{k}} m(d s) \cdot \liminf _{\nu \rightarrow \infty} \int_{G}\left|f\left(s^{-1} t\right)-\sum_{j} h_{j k}^{(\nu)}(s) g_{j k}^{(\nu)}(t)\right|^{2} m(d t)=0 ;
$$

also gilt für jedes $s$ ausserhalb einer $m^{*}$-Nullmenge $S$

$$
\liminf _{\nu \rightarrow \infty}\left\|T_{s} f-\sum_{j} h_{j k}^{(\nu)}(s) g_{j k}^{(\nu)}\right\|=0
$$

1) $\mu^{*}$ und zugehöriges $m^{*}$ brauchen nicht notwendig miteinander übereinzustimmen, wie man an einfachen Beispielen zeigen kann.

2) Siehe z.B. S. Kakutani: On the uniqueness of Haar's measure. Proc. 14 (1938), S. 27-31 ; K. Kodaira: [2], Kap. II.

3) K. Kodaira: [2], Satz 7.

4) K. Kodaira: [1], Satz 4; [2], Satz 22, a).

5) K. Kodaira: [2], Satz 12.

6) Hier ist es wesentlich, dass $m^{*}$ als das Lebesguesche Mass zweiter Art definiert ist. 
$T_{s} f$ gehört also für $s \in G-S$ zur durch $g_{j k}^{(\nu)}$ aufgespannte separable Mannigfaltigkeit $\mathfrak{M}$. Führt man die links-invariante "Quasi-metrik “ $\rho(s, t)=\left\|T_{s} f-T_{t} f\right\|$ ein, so ist $G-S$ also in bezug auf $\rho(s, t)$ separabel. Man setze nun $\theta_{\varepsilon}=\{t ; \rho(t, 1)<\varepsilon\}$. $\theta_{\varepsilon}$ ist dann $m^{*}$-messbar, und da $G-S \rho$-separabel ist, gibt es eine Folge $s_{1}, s_{2}, \ldots$ mit $G-S \subseteq \sum_{k} s_{k} \theta_{\epsilon}$. Es muss also $m\left(\theta_{\varepsilon}\right)>0$ sein. Hieraus folgt $s \theta_{\varepsilon} \leftarrow S$, also gibt es für jedes $s$ ein $t \in \theta_{\varepsilon}$ mit $s t \notin S$. Daher gilt

$$
\left\|T_{s} f-T_{s t} f\right\|=\left\|f-T_{t} f\right\|<\varepsilon,
$$

und dabei $T_{s t} f \in \mathfrak{M}$ ist. Für jedes $s \in S$ muss also $T_{s} f \in \mathfrak{M}$ sein.

Aus diesem Satz folgt der wichtige

Satz 6. Es seien höchstens abzählbar viele Bairesche Funktionen $f_{1}(t), f_{2}(t), \ldots$ auf $G$ gegeben. Dann gibt es einen bikompakten Normalteiler $N$ mit der separablen metrischen lokal kompakten Restklassengruppe $\bar{G}=G / N$, sodass jede $f_{k}(t)$ als eine Funktion auf $\bar{G}$ aufgefasst werden kann. Jede Aussage über die höchstens abzählbar vielen Baireschen Funktionen, die für jede separable, metrische, lokal kompakte Gruppe ${ }^{1)}$ richtig ist, gilt auch für allgemeine lokal bikompakte Gruppe.

Beweis. Es genügt den Fall $\mathrm{zu}$ betrachten, wo alle $f_{k}(t)$ stetig sind. Es sei $U$ eine so kleine Umgebung von 1 , dass $U U^{-1}$ relativ bikompakt ist, und $f_{0}(t)$ eine stetige Funktion mit

$$
f_{0}(t)=\left\{\begin{array}{lll}
1 & \text { für } & t=1, \\
0 & \text { für } & t \in G-U .
\end{array}\right.
$$

Ferner sei $\mathfrak{M}$ der durch alle $T_{s} f_{k}$ aufgespannte abgeschlossene lineare Teilraum von $\mathfrak{S}: \mathfrak{M}=\left[T_{s} f_{k} ; s \in G, k=0,1,2, \ldots\right]$, und $\mathfrak{N}=\mathfrak{E}-\mathfrak{M}$. $\quad G$ wird durch die Abbildung $s \rightarrow T_{s}$ in $\boldsymbol{U}$ topologisch isomorph eingebetett. $\mathfrak{M}$, und also $\mathfrak{R}$ sind aber $G$-invariant. Bezeichnet man die unitäre Transformationsgruppe von $\mathfrak{M}$ bzw. $\mathfrak{N}$ mit $\boldsymbol{U}_{\mathfrak{M}}$ bzw. $\boldsymbol{U}_{\mathfrak{N}}$, so lässt sich $G$ also als eine Untergruppe von $\boldsymbol{U}_{\mathfrak{M}} \times \boldsymbol{U}_{\mathfrak{R}}$ auffassen : man kann jedes $s \in G$ so schreiben :

$$
s=\bar{s} \times r, \quad \bar{s} \in \boldsymbol{U}_{\mathfrak{M}}, \quad r \in \boldsymbol{U}_{\mathfrak{n}} .
$$

Die aus alle $\bar{s}$ bestehende Untergruppe von $\boldsymbol{U}_{\mathfrak{m}}$ sei mit $\bar{G}$ bezeichnet. Nach dem obigen Satz ist $\mathfrak{M}$ separabel. Wählt man ein vollständiges orthonormiertes System $\left\{h_{1}, h_{2}, \ldots\right\}$ aus $\mathfrak{M}$, so ist $\bar{G}$ also eine separable metrische Gruppe mit der Metrik:

$$
\rho(\bar{s}, \bar{t})=\sum_{k=1}^{\infty} \frac{1}{2^{k}}\left\|T_{s} h_{k}-T_{t} h_{k}\right\| .
$$

Für jede Teilmenge $\bar{A} \subset \bar{G}$ ist offenbar

$$
A=\{s ; \bar{s} \in \bar{A}\}=G \cap\left(\bar{A} \times U_{\mathfrak{\Re}}\right) .
$$

$\bar{A}$ ist also dann und nur dann offen, wenn $A$ offen ist. Die Abbildung $s \rightarrow \bar{s}$ is also homomorph, stetig und gebietstreu. Setzt man $N=\{s$;

1) Unter einer metrischen Gruppe verstehen wir eine topologische Gruppe mit der links-invarianten Metrik. 
$\bar{s}=1\}$, so ist daher $\bar{G}=G / N$. Für genügend kleines $\varepsilon>0$ ist andererseits $\{s ; \rho(s, 1)<\varepsilon\}$ relativ bikompakt. Denn : aus

$$
\int_{G}\left|f_{0}\left(s^{-1} t\right)-f_{0}(t)\right|^{2} m(d t) \leqq\left\|f_{0}\right\|^{2}
$$

folgt $s \in U U^{-1}$. Hieraus folgt, dass $N$ bikompakt und $\bar{G}$ lokal-kompakt sind. Ferner ist für $s \in N f_{k}\left(s^{-1} t\right)=f_{k}(t)$, da $\int_{G}\left|f_{k}\left(s^{-1} t\right)-f_{k}(t)\right|^{2} m(d t)=$ $\left\|T_{s} f_{k}-f_{k}\right\|=0$ und $f_{k}(t)$ als stetig vorausgesetzt ist. Jedes $f_{k}(t)$ lässt sich also als eine Funktion auf $\bar{G}$ auffassen.

Für jede Teilmenge $\bar{A} \subset \bar{G}$ setzen wir nun

$$
\bar{m}^{*}(\bar{A})=m^{*}(A),
$$

wobei $A$ die zu $\bar{A}$ entsprechende Teilmenge von $\bar{G}$ bedeutet. Dann ist $\bar{m}^{*}$ das Haarsche Mass von $\bar{G}$. Denn : erstens ist es klar, dass $\bar{m}^{*}$ ein links-invariantes Carathéodorysches äusseres Mass in $\bar{G}$ ist und jede Borelsche Menge in $\bar{G} \bar{m}^{*}$-messbar ist; zweitens gilt

$$
\bar{m}^{*}(\bar{A})=\inf \bar{m}^{*}(\bar{U}), \quad \bar{U}: \text { offen und } \supset \bar{A},
$$

da für jede offene Menge $U \supset A$ die $\bar{U}=\{\bar{x} ; x N \subset U\} \supset \bar{A}$ offen ist und $\bar{m}^{*}(\bar{U}) \leqq m^{*}(U)$ genügt. Der über $N$ aufgenommene Mittelwert von einer stetigen Funktion $\varphi(u)$ auf $N$ sei mit $M \varphi(u)$ bezeichnet. Ist $\varphi(t)$ stetig auf $G$, so ist offenbar $\underset{u}{M} \varphi(u t)$ eine stetige Funktion auf $\bar{G}$. Normiert man nun das Haarsche Mass $n^{*}$ von $N$ sodass $n^{*}(N)=1$, so gilt für jede stetige Funktion $\varphi(t)$ auf $G$ nach dem Satz von Fubini

$$
\begin{aligned}
\int_{\bar{A} u} M \varphi(u t) \bar{m}(d \bar{t}) & =\int_{A} M \varphi(u t) m(d t)=\int_{A} m(d t) \int_{N} \varphi(u t) n(d u) \\
& =\int_{N} n(d u) \int_{A} \varphi(u t) m(d t)=\int_{A} \varphi(t) m(d t),
\end{aligned}
$$

wobei $\bar{A}$ eine $\bar{m}^{*}$-messbare Teilmenge von $\bar{G}$ und $A$ die zu $\bar{A}$ entsprechende Teilmenge von $G$ sind.

§4. Einzigkeit des Haarschsen Masses. Wir beweisen nun, dass das links-invariante Lebesguesche Mass erster Art auf $G$ mit dem zweiter Art, nämlich mit dem Haarschen Mass, übereinstimmt ${ }^{1}$. $\mathrm{Zu}$ diesem Zweck genügt es den folgenden Satz $\mathrm{zu}$ beweisen :

Satz 7. Jede offene Teilmenge von $G$ ist $m^{*}$-messbar.

Beweis. Es sei $U$ eine offene Teilmenge von $G$ mit $m^{*}(U)<+\infty$. Setzt man $m_{*}(U)=\sup _{B<U} m(B), B \in \mathfrak{b}(G),\left(m_{*}(U)\right.$ ist das $m^{*}$-innere Mass von $U$ ), so gibt es eine Bairesche Menge $B \subset U$ mit $m(B)=m_{*}(U)$. Nach dem Satz 6 kann man $B$ als eine Teilmenge von einer separablen metrischen Restklassengruppe $\bar{G}=\mathrm{G} / N$ nach einem bikompakten Normalteiler $N$ auffassen. Für jeden Punkt $s \in U-B$ konstruiere man nun eine stetige nicht negative Funktion $\varphi_{s}(t)$ mit

$$
\varphi_{s}(t)=\left\{\begin{array}{lll}
1 & \text { für } & t=s, \\
0 & \text { für } & t \in G-U,
\end{array}\right.
$$

1) Vgl. S. Kakutani : [1]. 
und setze $\bar{U}(\bar{s})=\left\{\bar{t} ; \underset{u}{M} \varphi_{s}(u t)>0\right\}, \bar{A}(s)=\bar{U}(\bar{s})-\bar{B}$. Dann gilt

$$
\int_{\bar{A}(s) u} M \varphi_{\varepsilon}(u t) \bar{m}(d \bar{t})=\int_{G-B} \varphi_{s}(t) m(d t)=0,
$$

da die Bairesche Menge $C=\left\{t ; \varphi_{s}(t)>0\right\}$ in $U$ enthalten ist und also nach $m(B)=m_{*}(U) \quad m(C-B)=0$ sein muss. $M \varphi_{s}(u t)$ ist andererseits in jedem $\bar{t} \in \bar{A}(s)$ positiv. Es ist also $\bar{m}(\bar{U}(\bar{s})-\bar{B})=\bar{m}(\bar{A}(s))=0 . \quad \bar{U}(\bar{s})$ ist offenbar eine Umgebung von $\bar{s} ; \bar{V}=\{\bar{s} ; s \in U-B\}$ wird also durch $\bar{U}(\bar{s})$ überdeckt. Wegen der Separabilität von $\bar{G}$ gibt es also unter $\bar{U}(\bar{s})$ höchstens abzählbar viele $\bar{U}\left(\bar{s}_{k}\right)$ mit $\bar{V} \subseteq \sum_{k} \bar{U}\left(\bar{s}_{k}\right)$. Setzt man nun $\bar{W}=\sum_{k}\left(\bar{U}\left(\bar{s}_{k}\right)-\bar{B}\right)$, so enthält die zu $\bar{W}$ entsprechende Teilmenge $W \subset G$ die Menge $U-B$ und es gilt $m^{*}(W)=\bar{m}(\bar{W}) \leqq \sum_{k} \bar{m}\left(\bar{U}\left(\bar{s}_{k}\right)-\bar{B}\right)$ $=0$. Es muss also $m^{*}(U)=m(B)$ sein, woraus die $m^{*}$-Messbarkeit von $U$ folgt. 